\title{
Bacillus spp. inoculation improves photosystem II efficiency and enhances photosynthesis in pepper plants
}

\author{
Blancka Yesenia Samaniego-Gámez ${ }^{1}$, René Garruña ${ }^{1^{*}}$, José M. Tun-Suárez ${ }^{1}$, \\ Jorge Kantun-Can ${ }^{1}$, Arturo Reyes-Ramirez ${ }^{1}$, and Lourdes Cervantes-Diaz ${ }^{2}$
}

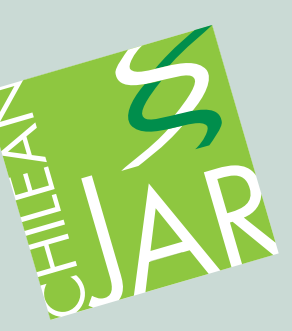

\section{ABSTRACT}

Bacillus is one of the main rhizobacteria to have been used as a study model for understanding many processes. However, their impact on photosynthetic metabolism has been poorly studied. The aim of this study was to evaluate the physiological parameters of pepper (Capsicum chinense Jacq.) plants inoculated with Bacillus spp. strains. Pepper seeds were inoculated with Bacillus cereus (K46 strain) and Bacillus spp. (M9 strain; a mixture of B. subtilis and $B$. amyloliquefaciens), chlorophyll fluorescence and gas exchange were evaluated. The ANOVA $(\mathrm{P} \leq 0.05)$ showed that the maximum photochemical quantum yield of photosystem II (PSII) $\left(F_{\mathrm{v}} / F_{\mathrm{m}}\right)$ in plants inoculated with the M9 strain (0.784) increased with respect to other treatments (K46: 0.744 and Control: 0.739). Inoculated plants with M9 and K46 strains exhibited an increase of both photochemical quenching (qP) (by $27 \%$ and $24 \%$, respectively) and $\mathrm{CO}_{2}$ assimilation rate (photosynthesis) (by $20 \%$ and $16 \%$, respectively), when compared with non-inoculated plants. Furthermore, plants inoculated with M9 and K46 showed decreased transpiration (61\% and $57 \%$, respectively) with respect to controls. Likewise, both electron transport rate of PSII (ETR) and PSII operating efficiency $\left(\Phi_{\text {PSII }}\right)$ increased in inoculated plants. However, only plants inoculated with the M9 strain showed enhancements on all growth characteristics. Our results therefore show that inoculating plants with M9 strain positively influenced the performance of the photosynthetic mechanism in pepper plants to increase chlorophyll fluorescence and gas exchange parameters. Promotion of photosynthetic capacity in pepper was due to increased ETR in the thylakoid membranes, which was promoted by the bacteria. M9 strain could even be used in sustainable agriculture programs.

Key words: Capsicum chinense, chlorophyll fluorescence, $\mathrm{CO}_{2}$ assimilation rate, plant growth promoting rhizobacteria.

${ }^{1}$ Instituto Tecnológico de Conkal, División de Estudios de Posgrado e Investigación, Av. Tecnológico s/n 97345, Conkal, Yucatán, México."Corresponding author (renegh10@hotmail.com).

${ }^{2}$ Universidad Autónoma de Baja California, Instituto de Ciencias Agrícolas, Carretera a Delta s/n 21705, Ejido Nuevo León, Baja California, México.

Received: 12 May 2016

Accepted: 10 November 2016

doi:10.4067/S0718-58392016000400003

\section{INTRODUCTION}

Plant growth promoting rhizobacteria (PGPR) are a diverse group of bacteria that can be found in the rhizosphere, on root surfaces and in association with roots (Ahemad and Kibret, 2014; Lucas et al., 2014). Rhizobacteria are proficient at colonizing the root surface, surviving, multiplying and competing with other microbiota, at least for the time needed to express their plant growth promotion/protection activities, which can improve plant fitness by different mechanisms (García-Cristobal et al., 2015). Directly, the presence of rhizobacteria can cause modifications to plant metabolism. Examples include $\mathrm{N}$ fixation, phosphate solubilization, Fe sequestration, and cytokinin, gibberellin, indoleacetic acid and ethylene production (Lucas et al., 2014). Indirectly, the presence of rhizobacteria promotes mechanisms that do not involve plant metabolism. Examples include antibiotics; lytic enzymes, such as chitinases, cellulases, 1,3-glucanases, proteases and lipases; siderophore production; competition between pathogens and non-pathogens; induced systemic resistance; and modulation of environmental stress effects (Ahemad and Kibret, 2014). Although the mechanisms used by rhizobacteria are well known, their impact on photosynthetic metabolism remains unclear (Shi et al., 2010; Stefan et al., 2013a; Palacios et al., 2014).

Some studies have reported that PGPR affect the physiology of plants to attenuate to some degree the stressful effects of drought, salt, UV, and a combination of high $\mathrm{CO}_{2}$ content and low atmospheric pressure (Burlak et al., 2013; Stefan et al., 2013b; García-Cristobal et al., 2015). Actually, some studies have shown that bacterial capacity to reduce reactive oxygen species (ROS) levels in drought stressed plants and were also correlated with an increase in photosynthesis (Armada et al., 2014). Thus, Shi et al. (2010) showed that endophytic bacteria species increased the photosynthetic capacity and total chlorophyll content of sugar beet, leading to a consequent increased carbohydrate synthesis, these increases were promoted by phytohormones (indole-3acetic acid, IAA) which were produced by the bacteria. The main parameters used to sensitively and objectively determine the ability of the strains to affect the photosynthetic apparatus in plants are minimum fluorescence $\left(\mathrm{F}_{0}\right)$, maximum photochemical quantum yield of photosystem II (PSII) $\left(F_{\mathrm{v}} / F_{\mathrm{m}}\right)$, PSII operating efficiency $\left(\Phi_{\mathrm{PSII}}\right)$, non-photochemical quenching (NPQ), and electron transport rate (ETR) (Lucas et al., 2014; García-Cristobal et al., 2015). Bacillus is one of the main rhizobacteria that has been used as a study model for understanding many processes, because it has been found to possess a wide genetic diversity adapted to different conditions and numerous properties of 
interest applied in industry, microbiology and agriculture (Niazi et al., 2014). Inoculation with B. subtilis increase photosynthesis in Arabidopsis through the modulation of plant endogenous sugar/abscisic acid (ABA) signaling, with a regulatory role for plant symbionts in photosynthesis (Zhang et al., 2008). However, Freitas et al. (2015) showed that iron accumulation in cassava was accompanied by an increase in the photosynthetic rate and biomass accumulation and may be linked with greater chlorophyll levels at this location. In this way, B. subtilis has been shown to increase the abundance of transcripts involved in iron uptake and transport as well as induce rhizosphere acidification that directly mobilizes soluble minerals (Zhang et al., 2009).

Furthermore, peppers (Capsicum spp.) are an economically important genus of the Solanaceae family and pepper plants are grown around the world because of their adaptation to different agro-climatic regions (Kraft et al., 2014). In particular, Habanero pepper (Capsicum chinense Jacq.) grows in wide temperature range, and has therefore been used in studies of plant stress (Garruña-Hernández et al., 2014; Valle-Gough et al., 2015).

Although studies have been performed on the effect of Bacillus in C. chinense (Kanchana et al., 2014; Gan et al., 2015), there are no previous studies on the effect of Bacillus spp. on the photosynthetic mechanisms of Capsicum spp. Therefore, two specific questions are addressed here: What are the physiological responses of $C$. chinense plants to inoculation with Bacillus spp. strains? Does photosynthesis increase in response to plant-rhizobacteria symbiosis? In order to contribute to the understanding of photosynthetic mechanisms during interactions between plants and rhizobacteria, the aim of this study was to evaluate the physiological parameters of $C$. chinense plants inoculated with Bacillus spp. strains.

\section{MATERIALS AND METHODS}

\section{Bacillus spp. strains and plant material}

Bacillus cereus (K46 strain) and Bacillus spp. (M9 strain; a mixture of $B$. subtilis and B. amyloliquefaciens) were isolated from soils in the state of Yucatán, Mexico. Strains were grown in $75 \mathrm{~mL}$ of nutrient broth under constant stirring at $200 \mathrm{rpm}$ for $96 \mathrm{~h}$ at $30{ }^{\circ} \mathrm{C}$. Subsequently, the cultures were centrifuged at $3500 \mathrm{rpm}$ for $15 \mathrm{~min}$ in a centrifuge (C-600 SOLBAT) at $29{ }^{\circ} \mathrm{C}$ to obtain the cells, which were washed twice with sterile saline $0.8 \%$ and dissolved in $2 \mathrm{~mL}$ saline for counting in a Neubauer chamber. The cell concentration was adjusted to $10^{8}$ cells $\mathrm{mL}^{-1}$ in $10 \mathrm{~mL}$ saline $8 \%$. Twenty seeds per tube were placed in a centrifuge for $1 \mathrm{~h}$ at $160 \mathrm{rpm}$.

The H241 genotype of Habanero pepper (Capsicum chinense Jacq.) was used, and seeds were disinfected with sodium hypochlorite $2 \%$ and washed three times with sterile distilled water. Sterile seeds were sown in germination trays with 200 cavities filled with sterile peat moss substrate moistened to field capacity with distilled water according to the experimental design. All trays were placed in a growth room at $20 \pm 2{ }^{\circ} \mathrm{C}$. They were watered every second day and leaf fertilized (UltraFol, Biochem, Constituyentes, México) weekly at a dose of $1 \mathrm{~g} \mathrm{~L}^{-1}$ distilled water. After $18 \mathrm{~d}$ germination, seedlings were transferred to $500 \mathrm{~mL}$ Styrofoam cups filled with sterile peat moss substrate. The seedlings were kept in a growth room at $20 \pm 2{ }^{\circ} \mathrm{C}$ and were watered and fertilized every second day as mentioned above. Ninety seedlings were randomly selected $60 \mathrm{~d}$ after seed sowing (DASS) and placed in a greenhouse.

\section{Chlorophyll fluorescence and gas exchange analyses}

The chlorophyll (Chl) fluorescence parameters were measured in vivo with a portable pulse amplitude modulation fluorometer (PAM Walz, Effeltrich, Germany). Measurements were performed $145 \mathrm{~d}$ after sowing at predawn $(05: 00 \mathrm{~h})$, on the third leaf from the shoot apex. The parameter $F_{\mathrm{v}} / F_{\mathrm{m}}$ represents the maximum photochemical quantum yield of PSII when reaction centers are open after dark adaptation, where $F_{\mathrm{v}}$ is maximum variable fluorescence (defined as $F_{\mathrm{m}}-F_{0}$ ), $F_{0}$ is minimum $\mathrm{Chl}$ fluorescence yield in the dark-adapted state, and $F_{\mathrm{m}}$ is maximum $\mathrm{Chl}$ fluorescence yield in the dark-adapted state. To determine the $F_{0}$ on excitation of leaf with a weak measuring beam. The $F_{\mathrm{m}}$ was determinated with a $0.6 \mathrm{~s}$ saturating pulse. After, an actinic light of $420 \mu \mathrm{mol}$ photons $\mathrm{m}^{-2} \mathrm{~s}^{-1}$ was switched on and saturating pulses $\left(8000 \mu \mathrm{mol}\right.$ photons $\left.\mathrm{m}^{-2} \mathrm{~s}^{-1}\right)$ were applied at $10 \mathrm{~min}$ intervals to determine the maximum fluorescence intensity in the light-adapted state $\left(F_{\mathrm{m}}{ }^{\prime}\right)$, minimum fluorescence intensity in the light-adapted state $\left(F_{0}{ }^{\prime}\right)$ and steady state fluorescence intensity in the light-adapted state $\left(F_{\mathrm{s}}\right)$ (Calatayud et al., 2002). The potential activity of PSII $\left(F_{\mathrm{v}} / F_{0}\right)$ was obtained according to Li et al. (2007). Both electron transport rate $\left(\mathrm{ETR}=\Phi_{\mathrm{PSII}} \times \mathrm{PAR} \times 0.5 \times\right.$ leaf absorptivity coefficient) and PSII operating efficiency $\left(\Phi_{\text {PSII }}\right.$ $\left.=\left(F_{\mathrm{m}}{ }^{\prime}-F_{\mathrm{s}}{ }^{\prime}\right) / F_{\mathrm{m}}{ }^{\prime}\right)$ were obtained through the application of a series of nine saturation pulses under increasing actinic irradiance (PAR) from 0 to $1500 \mu \mathrm{mol}$ photons $\mathrm{m}^{-2} \mathrm{~s}^{-1}$. The photochemical quenching $\left(\mathrm{qP}=\left(F_{\mathrm{m}}{ }^{\prime}-F_{\mathrm{s}}\right) /\left(F_{\mathrm{m}}{ }^{\prime}-F_{0}{ }^{\prime}\right)\right.$ and non-photochemical quenching $\left(\mathrm{NPQ}=F_{\mathrm{m}} / F_{\mathrm{m}}{ }^{\prime}-1\right)$ were calculated according to Kalaji et al. (2014).

$\mathrm{CO}_{2}$ assimilation rate $\left(\mathrm{A}_{\mathrm{N}}\right)$, stomatal conductance $\left(\mathrm{g}_{\mathrm{s}}\right)$ and transpiration (E) were measured using an infrared gas analyzer (IRGA; LICOR, LI-6400, Lincoln, Nebraska, USA). Water use efficiency (WUE) was calculated as $\mathrm{A}_{\mathrm{N}} / \mathrm{E}$ (Garruña-Hernández et al., 2014). Measurements were performed in the same leaves used in chlorophyll fluorescence measurement. The greenhouse conditions were $28{ }^{\circ} \mathrm{C}$, photosynthetic active radiation $1200 \mu \mathrm{mol}$ photons $\mathrm{m}^{-2} \mathrm{~s}^{-1}$, and $65 \% \mathrm{RH}$.

Total chlorophyll concentration was measured by SPAD502 portable chlorophyll meter (Minolta, Osaka, Japan) in the same leaves used previously. 


\section{Experimental design}

Thirty plants per treatment were used in a completely random design. The results were analyzed with one-way ANOVA and means were compared using Tukey's test at $\mathrm{P}$ $\leq 0.05$ (Statistica Six, StatSoft, Tulsa, Oklahoma, USA).

\section{RESULTS}

\section{Growth parameters and chlorophyll fluorescence parameters}

All treatments were significantly similar for plant height, but stem diameter, fresh weight and root volume of plants inoculated with Bacillus sp. M9 increased with respect to control plants by $22 \%, 34 \%$ and $58 \%$, respectively (Table 1 ).

The electron transport rate (ETR) of PSII increased in leaves of plants inoculated with M9 and K46 strains. ANOVA showed significant differences between treatments at above $420 \mu \mathrm{mol}$ photons $\mathrm{m}^{-2} \mathrm{~s}^{-1}$. At $1150 \mu \mathrm{mol}$ photons $\mathrm{m}^{-2} \mathrm{~s}^{-1}$ all treatments reached the maximum ETR (M9: 45.4; K46: 40.8 and control: 26.8) (Figure 1$)$. PSII operating efficiency $\left(\Phi_{\mathrm{PSII}}\right)$ was higher in inoculated plants than control plants from lowest to highest photosynthetic photon flux density (Figure 2). The maximum photochemical quantum yield of PSII $\left(F_{\mathrm{v}} / F_{\mathrm{m}}\right)$

Table 1. Growth parameters of Habanero pepper plants inoculated with Bacillus spp. (M9 and K46) and not inoculated (control).

\begin{tabular}{lcccc}
\hline Treatment & Plant height & Stem diameter & Fresh weight & Root volume \\
\hline & $\mathrm{cm}$ & $\mathrm{mm}$ & $\mathrm{g}$ & $\mathrm{mL}$ \\
M9 & $180 \pm 2.3$ & $27 \pm 1.7 \mathrm{a}$ & $1657 \pm 133 \mathrm{a}$ & $516 \pm 96 \mathrm{a}$ \\
K46 & $165 \pm 3.0$ & $20 \pm 1.1 \mathrm{ab}$ & $1051 \pm 64 \mathrm{~b}$ & $483 \pm 92 \mathrm{ab}$ \\
Control & $176 \pm 7.2$ & $21 \pm 2.1 \mathrm{~b}$ & $1093 \pm 106 \mathrm{~b}$ & $216 \pm 60 \mathrm{~b}$ \\
\hline
\end{tabular}

Data are means \pm SE. Different letters in the columns represent significant differences according to Tukey test $(\alpha=0.05)$.

Figure 1. Response curves of electron transport rate (ETR) to photosynthetic photon flux density in Habanero pepper plants inoculated with Bacillus spp. (M9 and K46) and not inoculated (control).

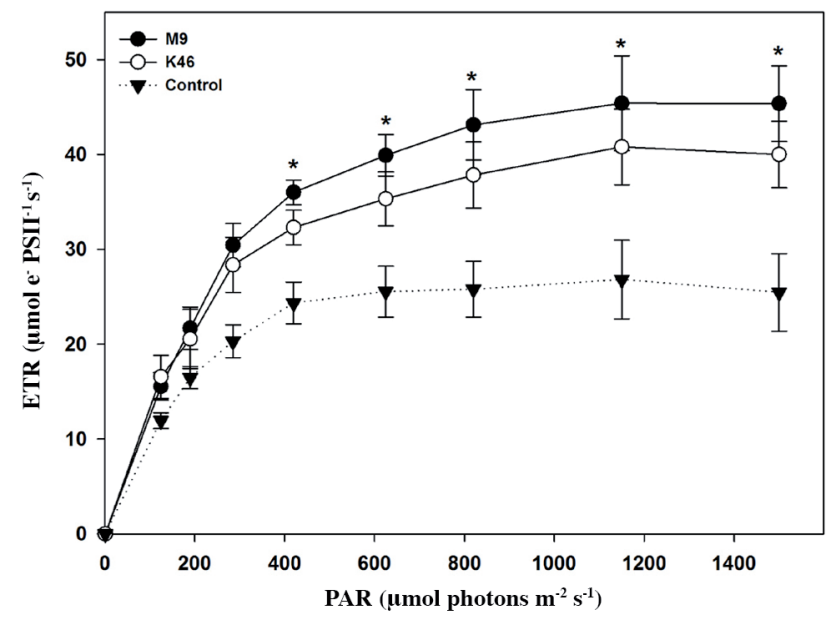

Data are means $\pm \mathrm{SE}$.

NS: nonsignificant, PSII: photosystem II.

*Significant between treatments at 0.05 probability level.
Figure 2. Photosystem II (PSII) operating efficiency ( $\left.\Phi_{\text {PSII }}\right)$ to photosynthetic photon flux density in Habanero pepper plants inoculated with Bacillus spp. (M9 and K46) and not inoculated (control).

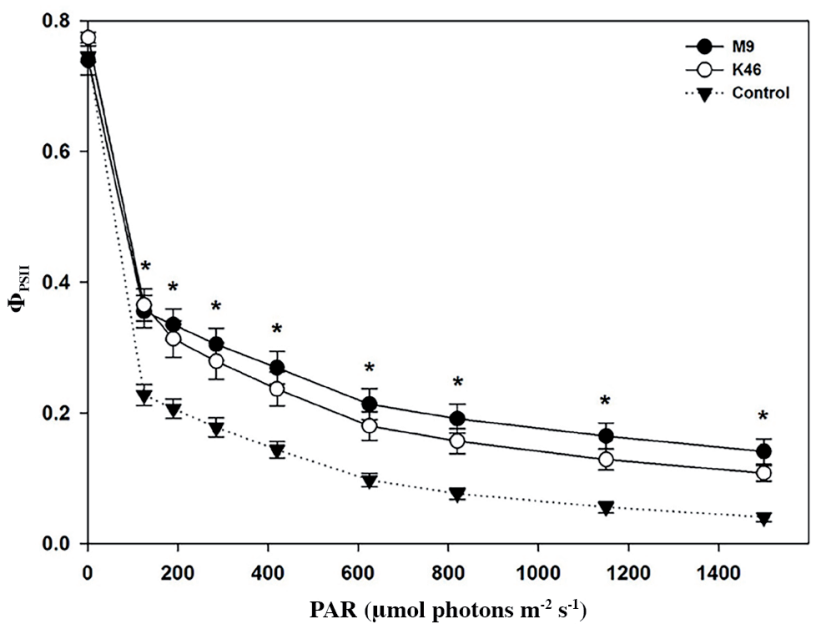

Data are means \pm SE. NS: nonsignificant. *Significant between treatments at 0.05 probability level.

of plants inoculated with M9 strain (0.784) increased with respect to plants inoculated with $\mathrm{K} 46$ (0.744) and control plants (0.739) (Figure 3A). On the other hand, the potential activity of PSII $\left(\mathrm{F}_{\mathrm{v}} / \mathrm{F}_{0}\right)$ showed a significantly similar trend to $F_{\mathrm{v}} / F_{\mathrm{m}}$. Values for plants inoculated with M9 strain (3.6) were $20 \%$ higher than for non-inoculated plants (2.9) (Figure 3B). Plants inoculated with M9 and K46 strains showed an increase in $\mathrm{qP}$ with respect to control plants $(27 \%$ and $24 \%$, respectively) (Figure 3C). Likewise, plants with M9 strain (1.18) increased the NPQ $26 \%$ with respect to control plants (0.88) (Figure 3D).

\section{Gas exchange analyses}

Inoculation with $\mathrm{M} 9$ and $\mathrm{K} 46$ increased the $\mathrm{CO}_{2}$ assimilation rate (photosynthesis) in Habanero pepper plants by $20 \%$ and $16 \%$, respectively, compared to non-inoculated plants (Figure 4A). Plants inoculated with M9 and K46 also showed decreases in both stomatal conductance $(56 \%$ and $45 \%$ respectively) (Figure $4 \mathrm{~B})$ and transpiration $(61 \%$ and $57 \%$ respectively) (Figure $4 \mathrm{C}$ ). However, the two strains improved WUE by $68 \%$ and $66 \%$ respectively (Figure 4D). On other hand, plants inoculated with the M9 increased SPAD values with respect to non-inoculated plants $(7 \%)$, but plants inoculated with the K46 had lower values than control plants (Figure 5).

\section{DISCUSSION}

Plant growth promoting rhizobacteria (PGPR) are known to favor plant growth via biological $\mathrm{N}$ fixation (Ashok et al., 2015), regulation of the synthesis of plant growth-regulators (Ahemad and Kibret, 2014) and solubilization of soilinsoluble compounds such as calcium di- and tri-phosphates and other minerals (Marschner, 2007). According to our results, inoculating plants with Bacillus spp. strains 
Figure 3. A) Maximum photochemical quantum yield of photosystem II (PSII) $\left(F_{\mathrm{v}} / F_{\mathrm{m}}\right)$, B) potential activity of PSII $\left(\boldsymbol{F}_{\mathrm{v}} / \boldsymbol{F}_{0}\right)$, C) photochemical quenching (qP) and D) non-photochemical quenching (NPQ) in Habanero pepper plants inoculated with Bacillus spp. (M9 and K46) and not inoculated (control).

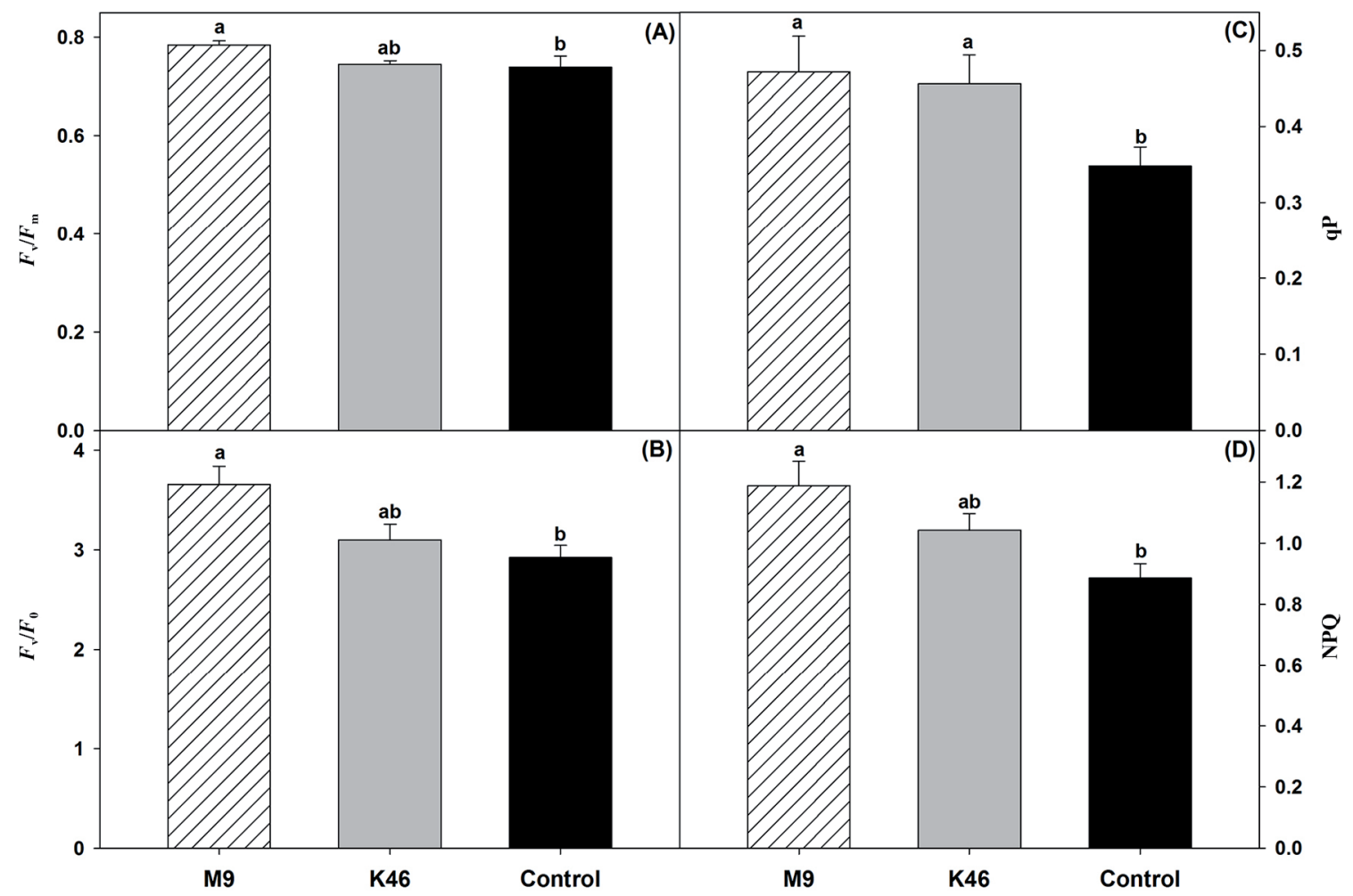

Actinic light intensity: $420 \mu \mathrm{mol} \mathrm{m} \mathrm{s}^{-2}$.

Data are means \pm SE. Different letters represent significant differences between treatments according to Tukey test $(\alpha=0.05)$.

modified the physiological mechanisms with respect to control plants (not inoculated).

The electron transport rate (ETR) of PSII increased in leaves of plants inoculated with Bacillus spp. strains. According to Li et al. (2007), the ETR of PSII is inhibited by stress. However, the plants in this study were not subjected to stressful conditions. Therefore, the increase in ETR values is a consequence of the positive effect of PGPR. According to Melis (1999), an increase in the electron transport rate of PSII suggests that the quinone acceptor $\left(Q_{a}\right)$ is highly oxidized and its excitation energy is utilized in electron transport, thereby avoiding photodamage. Inoculating plants therefore has the potential to increase photosynthesis rates and plant growth.

Additionally, it is observed that PSII operating efficiency $\left(\Phi_{\text {PSII }}\right)$ increased in inoculated plants. This parameter measures the proportion of light absorbed by chlorophyll associated with PSII that is used in photochemistry (Maxwell and Johnson, 2000). Habanero pepper plants inoculated with Bacillus spp. strains therefore showed an increase in overall photosynthetic capacity in vivo. Zhang et al. (2007) showed that certain PGPR elevate photosynthesis in Arabidopsis through the modulation of endogenous sugar/abscisic acid (ABA) signaling and establish a regulatory role for soil symbionts in plant energy acquisition. Some Bacillus are known to possess volatile organic compounds that function as modulators, sensing primary and secondary metabolism, stress responses and hormone regulation in plants (Zhang et al., 2007).

Moreover, the maximum photochemical quantum yield of PSII $\left(F_{\mathrm{v}} / F_{\mathrm{m}}\right)$ increased in inoculated plants. According to Baker (2008), $F_{\mathrm{v}} / F_{\mathrm{m}}$ is the maximum efficiency at which light absorbed by PSII is used for reduction of $\mathrm{Q}_{\mathrm{A}}$, and is used as a sensitive indicator of plant photosynthetic performance. An $F_{\mathrm{v}} / F_{\mathrm{m}}$ value of around 0.83 is an optimal measurement for most plant species (Maxwell and Johnson, 2000). This suggests that the M9 strain promoted a healthy PSII in inoculated plants, and other authors obtained similar results (Gururani et al., 2012), with increased $F_{\mathrm{v}} / F_{\mathrm{m}}$ values when using PGPR.

On other hand, the potential activity of PSII $\left(\mathrm{F}_{\mathrm{v}} / \mathrm{F}_{0}\right)$ showed a similar trend to $F_{\mathrm{v}} / F_{\mathrm{m}}$. Li et al. (2007) found a decrease of $21.7 \%$ in $\mathrm{F}_{\mathrm{v}} / \mathrm{F}_{0}$ after $48 \mathrm{~h}$ water stress in Medicago sativa. Yang et al. (2008) mentioned that PGPR also elicit so-called 'induced systemic tolerance' to salt and drought. Our results therefore showed that inoculation with PGPR could prepare the plant for future abiotic stress situations.

Plants inoculated with M9 and K46 had an increase in $\mathrm{qP}$ with respect to control plants; $\mathrm{qP}$ denotes the proportion of excitons captured by open traps and being converted to chemical energy in the PSII reaction center (Krause and Weis, 1991). 
Figure 4. A) $\mathrm{CO}_{2}$ Assimilation rate $\left(\mathrm{A}_{\mathrm{N}}\right)$, B) stomatal conductance $\left(\mathrm{g}_{\mathrm{s}}\right)$, C) transpiration (E), and D) water use efficiency (WUE) of Habanero pepper plants inoculated with Bacillus spp. (M9 and K46) and not inoculated (control).

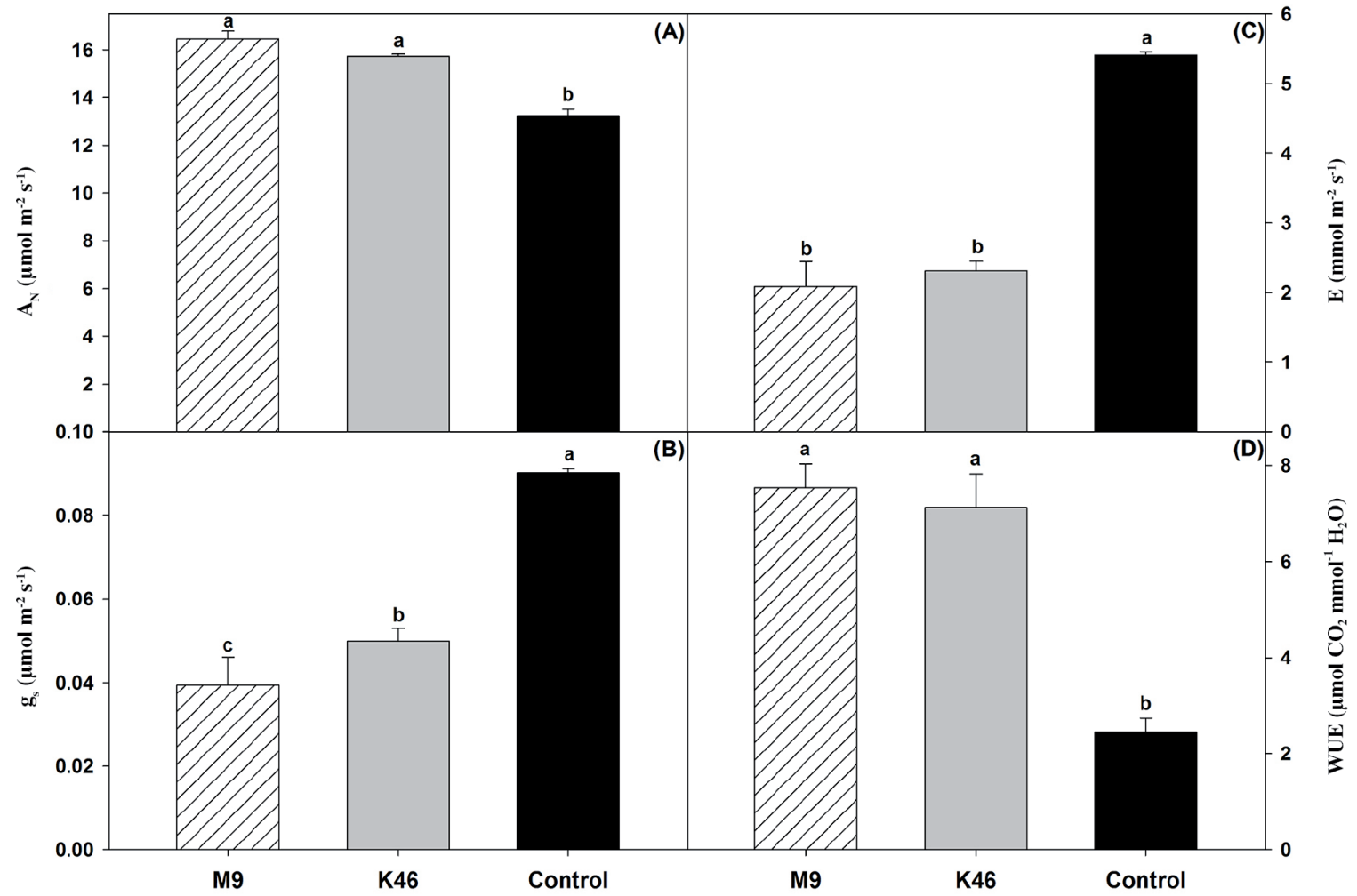

Data are means \pm SE. Different letters represent significant differences between treatments according to Tukey test $(\alpha=0.05)$.

Figure 5. SPAD values of Habanero pepper plants inoculated with Bacillus spp. (M9 and K46) and not inoculated (control).

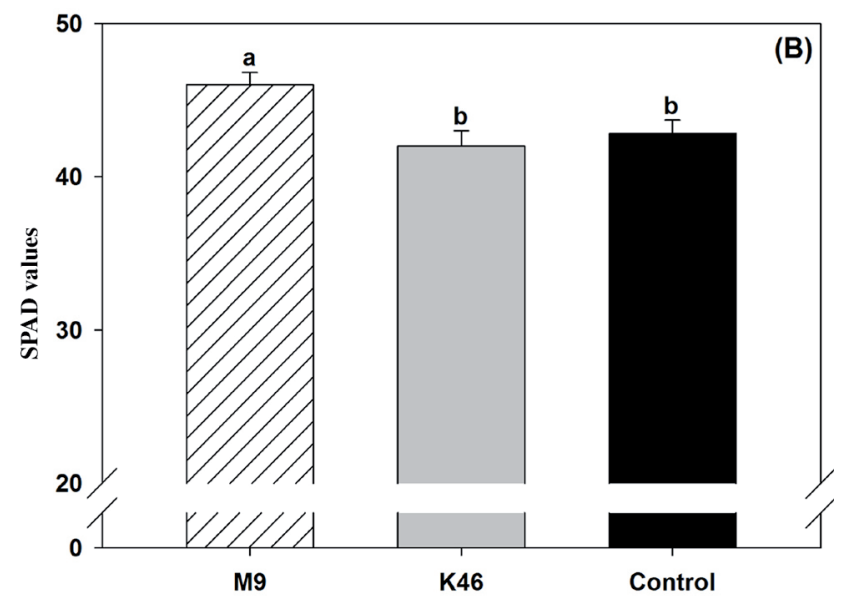

Data are means \pm SE. Different letters represent significant differences between treatments according to Tukey test $(\alpha=0.05)$.

Likewise, NPQ was higher in plants inoculated. This study therefore showed that inoculated plants had a better way of getting rid of excess light energy that had already been absorbed, because of the non-photochemical mechanisms that quench singlet-excited chlorophylls (Chl) and harmlessly dissipate excess excitation energy as heat (Müller et al., 2001). Both qP and NPQ help to minimize production of $\mathrm{O}_{2}$ in the PSII antenna (Müller et al., 2001).
On the other hand, our results therefore show that plants inoculated with Bacillus strains modified gas exchange to enhance $\mathrm{CO}_{2}$ assimilation (photosynthesis) and reduce water release by leaves. Water use efficiency in inoculated plants was therefore better, assimilating more $\mathrm{CO}_{2}$ molecules per $\mathrm{H}_{2} \mathrm{O}$ molecule released by stomata. Some authors (Vivas et al., 2003; Vardharajula et al., 2011; Armada et al., 2015) mention that plants inoculated with PGPR developed drought tolerance. According to Kumar et al. (2015), PGPR influence the physiology of the whole plant and play a key role in plant growth and the yield of various crops. Plants are known to release part of their photosynthetic output in the form of root exudates that attract and maintain bacterial colonies in the soil, promoting mutually beneficial effects, including the enhancement of plant abiotic stress tolerance by PGPR (Liu and Zhang, 2015).

Additionally plants inoculated with M9 increased SPAD values with respect to non-inoculated plants. MarulandaAguirre et al. (2008) observed decreased photosynthetic pigments (chlorophyll and carotenoids) in Lactuca sativa plants inoculated with Bacillus megaterium, but photosynthetic pigments increased when $B$. megaterium was combined with an arbuscular mycorrhizal fungus. The effect of PGPR on photosynthetic pigments may therefore depend on the strain used for inoculation.

Moreover, it was observed that the inoculation with the Bacillus spp. M9, increased plant growth. Inoculation 
with PGPR was found to improve plant growth in previous studies on pepper plants, Kang et al. (2007) used Pseudomonas rhodesiae and Pantoea ananatis and promoted significant growth of peppers, enhancing their root fresh weight by $73.9 \%$ and $41.5 \%$, respectively. Datta et al. (2011) found that the inoculation of pepper plants with strains of Bacillus spp. and Streptomyces spp. increased growth characteristics such as total number of fruits, fruit weight and yield. Luna et al. (2013) evaluated pepper plants inoculated with Bacillus spp. strains and observed an increase in biomass. There are different mechanisms explaining how PGPR favor plant growth: Biological N fixation (Ashok et al., 2015); regulation of the synthesis of plant growth-regulators, which can promote root growth and proliferation of root hairs, enhancing the uptake of water and minerals from the soil (Ahemad and Kibret, 2014); and a decrease in ethylene content of the developing or stressed plants, inducing elongation of the root system (Vessey, 2003).

Although some physiological parameters increased with K46 strain, plant growth parameters were similar from those of controls. According with Ahemad and Kibret (2014) the plant-beneficial symbiotic interactions are influenced by root exudates, composition of these exudates is dependent upon the physiological status and species of plants and microorganisms, probably K46 strain has not an excellent synergy with pepper plants, and its increase in photosynthesis was not enough to increase biomass. However, all plant growth parameters improved in pepper plants inoculated with M9 strain with respect to control plants. The microorganisms can promote plant growth by regulating nutritional and hormonal balance, producing plant growth regulators and solubilizing nutrients (Nadeem et al., 2014). According to Patten and Glick (1996) the microorganisms isolated from rhizosphere synthesize auxins (indole-3-acetic acid/indole acetic acid/IAA). The IAA affects plant cell division, pigment formation and photosynthesis by changing the plant auxin pool (Ahemad and Kibret, 2014). Moreover, $\mathrm{CO}_{2}$ resulting from respiration of bacteria can help to increase photosynthesis. Hibberd and Quick (2002) reported that $\mathrm{CO}_{2}$ produced in roots can be transported to the shoot; stem cells in tobacco are supplied with $\mathrm{C}$ for photosynthesis from the vascular system and not from stomata. In this way, Rozpadek et al. (2015) suggest that upon endophyte colonization, host plant undergoes changes in its photosynthetic apparatus, leading to increased light harvesting and photosynthesis efficiency.

Here, M9 strain improved chlorophyll fluorescence parameters and total chlorophyll concentration, suggesting more reaction centers and higher light harvesting; thus, the quinone acceptor $\left(\mathrm{Q}_{\mathrm{a}}\right)$ is highly oxidized and its excitation energy is utilized in electron transport, leading higher ATP and NADPH production, employed for $\mathrm{C}$ assimilation in the Calvin cycle, and improving plant growth.

\section{CONCLUSION}

Based on our results, we concluded that M9 strain (Bacillus spp.) used in this study demonstrated a striking influence on all parameters evaluated. Both strains (M9 and K46) improved the efficiency of photosystem II (PSII) to increase both the electron transport rate (ETR) and PSII operating efficiency $\left(\Phi_{\text {PSII }}\right)$ of inoculated plants. However, M9 increased photochemical quenching (qP), nonphotochemical quenching (NPQ), maximum photochemical quantum yield (fluorescence) and potential activity of PSII. In addition, plants inoculated with M9 and K46 showed remarkably improved gas exchange, increasing both the net assimilation rate (photosynthesis) and water use efficiency (WUE), and decreasing transpiration rate. Inoculation with Bacillus spp. (M9) and B. cereus (K46) therefore positively influenced the performance of the photosynthetic mechanism in Habanero pepper plants. However, plants inoculated with the M9 strain showed enhancements on all growth characteristics. M9 strain could be used in sustainable agriculture programs.

\section{ACKNOWLEDGEMENTS}

Thank to Consejo Nacional de Ciencia y Tecnología (CONACYT) for the scholarship \#253611 for doctoral studies by B.Y. Samaniego-Gámez and for master's grant \#334212 to J. Kantun-Can.

\section{REFERENCES}

Ahemad, M., and M. Kibret. 2014. Mechanisms and applications of plant growth promoting rhizobacteria: Current perspective. Journal of King Saud University 26:1-20.

Armada, E., J.M. Barea, P. Castillo, A. Roldán, and R. Azcón. 2015. Characterization and management of autochthonous bacterial strains from semiarid soils of Spain and their interactions with fermented agrowastes to improve drought tolerance in native shrub species. Plant Physiology and Biochemistry 96:306-318.

Armada, E., G. Portela, A. Roldán, and R. Azcón. 2014. Combined use of beneficial soil microorganism and agrowaste residue to cope with plant water limitation under semiarid conditions. Geoderma 232:640-648.

Ashok, K.A., I. Bahadur, B.R. Maurya, R. Raghuwanshi, V.S Meena, D.K. Singh, et al. 2015. Does a plant growth promoting rhizobacteria enhance agricultural sustainability? Journal of Pure and Applied Microbiology 9:715-724.

Baker, N.R. 2008. Chlorophyll fluorescence: a probe of photosynthesis in vivo. Annual Review of Plant Biology 59:89-113.

Burlak, O.P., J.P. de Vera, V. Yatsenko, and N.O. Kozyrovska. 2013. Putative mechanisms of bacterial effects on plant photosystem under stress. Biopolymers and Cell 29:3-10.

Calatayud, A., J.W. Ramirez, D.J. Iglesias, and E. Barreno. 2002. Effects of ozone on photosynthetic $\mathrm{CO}_{2}$ exchange, chlorophyll a fluorescence and antioxidant systems in lettuce leaves. Physiologia Plantarum 116:308-316. 
Datta, M., R. Palit, C. Sengupta, M.K. Pandit, and S. Banerjee. 2011. Plant growth promoting rhizobacteria enhance growth and yield of chilli (Capsicum annuum L.) under field conditions. Australian Journal of Crop Science 5:531-536.

Freitas, M.A., F.H. Medeiros, S.P. Carvalho, L.R. Guilherme, W.D. Teixeira, H. Zhang, et al. 2015. Augmenting iron accumulation in cassava by the beneficial soil bacterium Bacillus subtilis (GB03). Frontiers in Plant Science 6:596. doi:10.3389/ fpls.2015.00596.

Gan, H.Y., H.M. Gan, M.A. Savka, A.J. Triassi, M.S. Wheatley, K.F. Naqvi, et al. 2015. Whole-genome sequencing and annotation of Bacillus safensis RIT372 and Pseudomonas oryzihabitans RIT370 from Capsicum annuum (bird's eye chili) and Capsicum chinense (yellow lantern chili), respectively. Genome Announcements 3:e00288-15.

García-Cristobal, J., A. García-Villaraco, B. Ramos, J. GutierrezMañero, and J.A. Lucas. 2015. Priming of pathogenesis relatedproteins and enzymes related to oxidative stress by plant growth promoting rhizobacteria on rice plants upon abiotic and biotic stress challenge. Journal of Plant Physiology 188:72-79.

Garruña-Hernández, R., R. Orellana, A. Larque-Saavedra, and A. Canto. 2014. Understanding the physiological responses of a tropical crop (Capsicum chinense Jacq.) at high temperature. Public Library of Science ONE 9(11):e111402. doi:10.1371/ journal.pone.0111402.

Gururani, M.A., C.P. Upadhyaya, V. Baskar, J. Venkatesh, A. Nookaraju, and S.W. Park. 2012. Plant growth-promoting rhizobacteria enhance abiotic stress tolerance in Solanum tuberosum through inducing changes in the expression of ROSscavenging enzymes and improved photosynthetic performance. Journal of Plant Growth Regulation 32:245-258.

Hibberd, J.M., and W.P. Quick. 2002. Characteristics of C-4 photosynthesis in stems and petioles of $\mathrm{C}-3$ flowering plants. Nature 415:451-454.

Kalaji, H.M., G. Schansker, R.J. Ladle, V. Goltsev, K. Bosa, S.I. Allakhverdiev, et al. 2014. Frequently asked questions about in vivo chlorophyll fluorescence: practical issues. Photosynthesis Research 122:121-158.

Kanchana, D., M. Jayanthi, G. Usharani, P. Saranraj, and D. Sujitha. 2014. Interaction effect of combined inoculation of PGPR on growth and yield parameters of Chilli Var K1 (Capsicum annuum L.) International Journal of Microbiology Research 5:144-151.

Kang, S.H., H.S. Cho, H. Cheong, C.M. Ryu, J.F. Kim, and S.H. Park. 2007. Two bacterial endophytes eliciting boot plant growth promotion and plant defense on pepper (Capsicum annuиm L.) Journal of Microbiology and Biotechnology 17:96-103.

Kraft, K.H., C.H. Brown, G.P. Nabhan, E. Luedeling, J.J. LunaRuiz, G. Coppens d'Eeckenbrugge, et al. 2014. Multiple lines of evidence for the origin of domesticated chili pepper, Capsicum annuum, in Mexico. Proceedings of the National Academy of Sciences of the United States of America 111(17):6165-6170. doi/10.1073/pnas.1308933111.

Krause, G.H., and E.E. Weis. 1991. Chlorophyll fluorescence and photosynthesis: The basics. Annual Review of Plant Physiology and Plant Molecular Biology 42:313-49.

Kumar, S., M. Agarwal, S. Dheeman, and D.K. Maheshwari. 2015. Exploitation of phytohormone-producing PGPR in development of multispecies bioinoculant formulation. p. 297-317. In Maheshwari, D.K. (ed.) Bacterial metabolites in sustainable agroecosystem, sustainable development and biodiversity. Vol. 12. Springer International Publishing, Cham, Switzerland.
Li, W., S. Zhang, and L. Shan. 2007. Responsibility of nonstomatal limitations for the reduction of photosynthesis-response of photosynthesis and antioxidant enzyme characteristics in alfalfa (Medicago sativa L.) seedlings to water stress and rehydration. Frontiers of Agriculture in China 1:265-264.

Liu, X.M., and H. Zhang. 2015. The effects of bacterial volatile emissions on plant abiotic stress tolerance. Frontiers in Plant Science 6:774.

Lucas, J.A., J. García-Cristobal, A. Bonilla, B. Ramos, and J. Gutierrez-Mañero. 2014. Beneficial rhizobacteria from rice rhizosphere confers high protection against biotic and abiotic stress inducing systemic resistance in rice seedlings. Plant Physiology and Biochemistry 82:44-53.

Luna, M.L., P.R.A. Martínez, I.M. Hernández, M.S.M. Arvizu, y A.J.R. Pacheco. 2013. Caracterización de rizobacterias aisladas de tomate y su efecto en el crecimiento de tomate y pimiento. Revista Fitotecnia Mexicana 36:63-69.

Marschner, P. 2007. Plant-microbe interactions in the rhizosphere and nutrient cycling. p. 159-182. In Marschner, P., and Z. Rengel (eds.) Soil biology, nutrient cycling in terrestrial ecosystems. Vol. 10. Springer-Verlag, Berlin, Heidelberg, Germany.

Marulanda-Aguirre, A., R. Azcón, J.M. Ruiz-Lozano, and R. Aroca. 2008. Differential effects of a Bacillus megaterium strain on Lactuca sativa plant growth depending on the origin of the arbuscular mycorrhizal fungus coinoculated: physiologic and biochemical traits. Journal of Plant Growth Regulation 27:10-18.

Maxwell, K., and G.N. Johnson. 2000. Chlorophyll fluorescence-a practical guide. Journal of Experimental Botany 51:659-668.

Melis, A. 1999. Photosystem-II damage and repair cycle in chloroplasts: what modulates the rate of photodamage in vivo? Trends in Plant Sciences 4:1360-1385.

Müller, P., X.P. Li, and K.K. Niyogi. 2001. Non-photochemical quenching. A response to excess light energy. Plant Physiology 125:1558-1566

Nadeem, S.M., M. Ahmad, Z.A. Zahir, A. Javaid, and M. Ashraf. 2014. The role of mycorrhizae and plant growth promoting rhizobacteria (PGPR) in improving crop productivity under stressful environments. Biotechnology Advances 32:429-448.

Niazi, A.A., S. Manzoor, S. Asari, S. Bejai, J. Meijer, and E. Bongcam-Rudloff. 2014. Genome analysis of Bacillus amyloliquefaciens subsp. plantarum UCMB5113: a rhizobacterium that improves plant growth and stress management. Public Library of Science ONE e104651. doi:10.1371/journal.pone.0104651.

Palacios, O.A., Y. Bashan, and L.E. de-Bashan. 2014. Proven and potential involvement of vitamins in interactions of plants with plant growth-promoting bacteria- an overview. Biology and Fertility of Soils 50:415-432.

Patten, C.L., and B.R. Glick. 1996. Bacterial biosynthesis of indole3-acetic acid. Canadian Journal of Microbiology 42:207-220.

Rozpadek, P., K. Wężowicz, M. Nosek, R. Ważny, K. Tokarz, M. Lembicz, et al. 2015. The fungal endophyte Epichloë typhina improves photosynthesis efficiency of its host orchard grass (Dactylis glomerata). Planta 242:1025-1035.

Shi, Y., K. Lou, and C. Li. 2010. Growth and photosynthetic efficiency promotion of sugar beet (Beta vulgaris L.) by endophytic bacteria. Photosynthesis Research 105:5-13.

Stefan, M., N. Munteanu, V. Stoleru, and M. Mihasan 2013a. Effects of inoculation with plant growth promoting rhizobacteria on photosynthesis, antioxidant status and yield of runner bean. Romanian Biotechnological Letters 18:8132-8143. 
Stefan, M., N. Munteanu, V. Stoleru, M. Mihasan, and L. Hritcu. 2013b. Seed inoculation with plant growth promoting rhizobacteria enhances photosynthesis and yield of runner bean (Phaseolus coccineus L.) Scientia Horticulturae 151:22-29.

Valle-Gough, R.E., S.A. Avilés-Viñas, S. López-Erosa, A. CantoFlick, E. Gómez-Uc, L.A. Sáenz-Carbonell, et al. 2015. Polyamines and WOX genes in the recalcitrance to plant conversion of somatic embryos of Habanero pepper (Capsicum chinense Jacq.) African Journal of Biotechnology 14:569-581.

Vardharajula, S., A.Z. Ali, M. Grover, G. Reddy, and V. Bandi. 2011. Drought-tolerant plant growth promoting Bacillus spp.: effect on growth, osmolytes, and antioxidant status of maize under drought stress. Journal of Plant Interactions 6:1-14.

Vessey, K.J. 2003. Plant growth promoting rhizobacteria as biofertilizers. Plant and Soil 255:571-586.

Vivas, A., A. Marulanda, J.M. Ruiz-Lozano, J.M. Barea, and R. Azcón. 2003. Influence of a Bacillus sp. on physiological activities of two arbuscular mycorrhizal fungi and on plant responses to PEG-induced drought stress. Mycorrhiza 13:249-256.
Yang, J., J.W. Kloepper, and C.M. Ryu. 2008. Rhizosphere bacteria help plants tolerate abiotic stress. Trends in Plant Sciences 14:1360-1385.

Zhang, H., M.S. Kim, V. Krishnamachari, P. Payton, Y. Sun, M. Grimson, et al. 2007. Rhizobacterial volatile emissions regulate auxin homeostasis and cell expansion in Arabidopsis. Planta 226:839-851

Zhang, H., Y. Sun, X. Xie, M.S. Kim, S.E. Dowd, and P.W. Paré. 2009. A soil bacterium regulates plant acquisition of iron via deficiency inducible mechanisms. Plant Journal 58:568-577.

Zhang, H., X. Xie, M.S. Kim, D.A. Kornyeyev, S. Holaday, and P.W. Pare. 2008. Soil bacteria augment Arabidopsis photosynthesis by decreasing glucose sensing and abscisic acid levels in planta. Plant Journal 56:264-273. 